\title{
Self-healing thermoplastic elastomer brush copolymers having a glassy polymethylmethacrylate backbone and rubbery polyacrylate-amide brushes
}

\author{
Yulin Chen, Zhibin Guan* \\ Department of Chemistry, University of California, 1102 Natural Sciences 2, Irvine, CA 92697, \\ USA. \\ *Corresponding author. \\ Fax: +1 9498242210; Tel: +1 9498245172; E-mail: zguan@uci.edu
}

\begin{abstract}
We synthesized a series of brush copolymers having glassy polymethylmethacrylate (PMMA) backbone and flexible polyacrylate-amide (PA-amide) brushes that exhibit thermoplastic elastomer properties. Importantly, the dynamic hydrogen bonds in the soft PA-amide matrix enables the material to self-heal after mechanical damage at room temperature without the need of any external stimulus.
\end{abstract}

Keywords: self-healing materials, brush copolymers, thermoplastic elastomers, multiphase materials 


\section{Introduction}

Self-healing materials have emerged as promising next-generation materials in response to the concerns about the sustainability and growing environmental issues. Introducing self-healing capability into materials would make them more adaptable, durable and self-maintainable [1-5]. Following the pioneering work using microcapsulated healing agent for self-healing design [6,7], many approaches have been reported to prepare materials that can self-heal, including the employment of reversible [8-12] and irreversible [13,14] chemical reactions, metal-ligand supramolecular interaction $[15,16], \pi-\pi$ stacking [17,18], and hydrogen-bonding [19-22]. Based on healing mechanisms, self-healing polymers are broadly classified into extrinsic and intrinsic systems. While extrinsic systems require special healing agents for healing [6], intrinsic systems rely on intrinsically built-in dynamic molecular interactions to enable the healing process. Currently, many of the intrinsic self-healing material systems require the input of external energy (e.g., heat [8] or light [15]). For practical applications, it is desirable to develop intrinsic systems that can spontaneously heal without external intervention. To achieve such intrinsic self-healing materials, dynamic, reversible molecular interactions are generally required for the design [23]. However, one major challenge with this approach is that if a material is composed of reversible, highly dynamic molecular interactions, the mechanical properties are generally weak [24].

We recently demonstrated a multiphase approach to address this challenge in designing selfhealing polymers utilizing non-covalent, dynamic interactions [22]. Specifically, we circumvent the inherent trade-off between stiff low-strain mechanical response and ambient, stimulus-free dynamic self-healing by introducing a discontinuous glassy hard phase (to improve stiffness and

yield strength) covalently connected to a continuous soft phase bearing a robust network of $\mathrm{H}-$ 
bonding dynamic motifs to effect nearly quantitative self-healing after catastrophic macroscopic damage. In our initial demonstration, we designed a self-healing thermoplastic elastomer (TPE) employing brush copolymers composed of the glassy polystyrene (PS) backbone grafted with multiple brushes of the highly dynamic polyacrylate-amide (PA-amide) chains [22]. The system microphase separates into two domains with the glassy PS backbone providing the stiffness and strength for the overall bulk materials, while the amide groups on the brushes served as the selfhealing motifs through the reversible hydrogen-bonding interaction. Based on this multiphase design, self-healing materials from core-shell nanoparticles [25], block copolymers [16, 26, 27] were developed.

In a recent study, we prepared a series of triblock copolymers having PMMA central block flanked by two PA-amide terminal blocks [27]. The polymers form hard/soft two-phase systems with a combination of excellent mechanical properties and self-healing capability. Compared to our initially reported PS brush copolymer system [22], the PMMA triblock copolymer system showed improved mechanical properties in terms of higher Young's modulus and tensile strength while maintaining good self-healing capability. The enhanced mechanical properties could potentially be due to stronger interactions between the soft PA-amide and hard PMMA microphases as both contain polar ester groups. In current study, we want to investigate a selfhealing polymer system having PMMA hard domain and PA-amide soft phase in a brush macromolecular architecture (Scheme 1). For this purpose, PMMA macroinitiators were first synthesized by free radical polymerization (Scheme 1a). The PA-amide was grafted onto the PMMA backbone by atom transfer radical polymerization (ATRP) (Scheme 1b). The synthesized 
polymers were fully characterized and the mechanical properties and self-healing capability of the bulk materials were evaluated.

a.

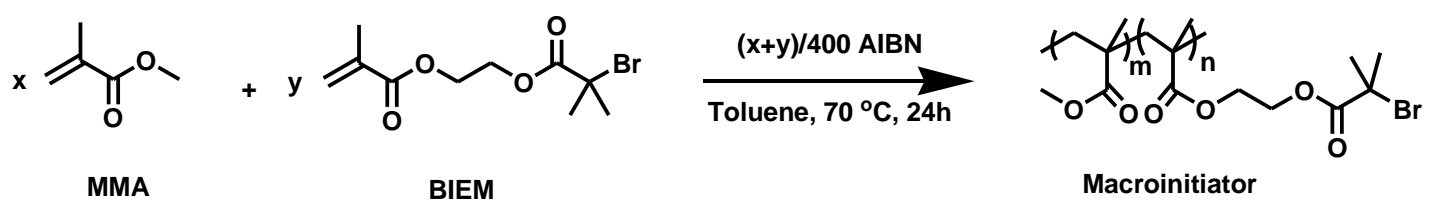

b.

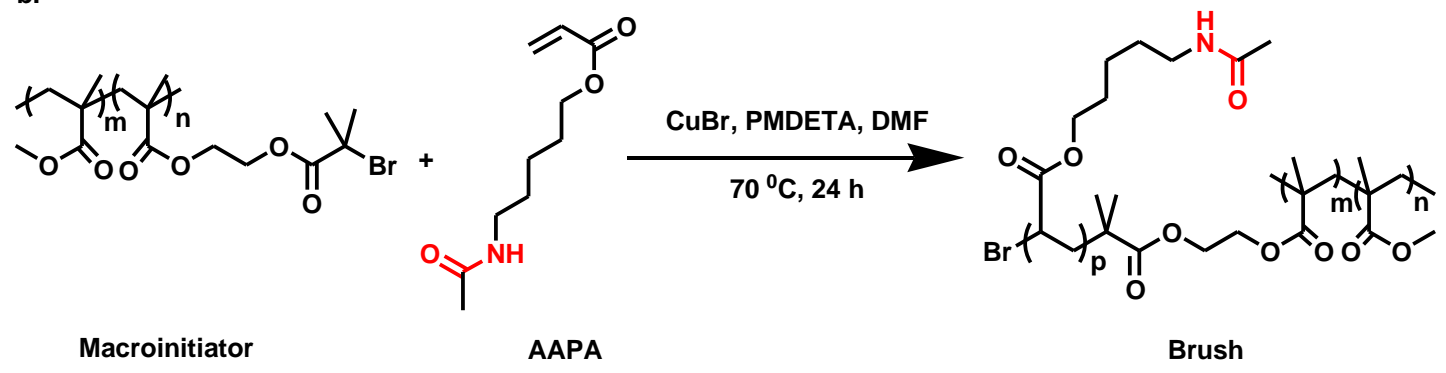

Scheme 1. Synthesis of the self-healing brush copolymers. a) synthesis of macroinitiators by free radical polymerization. b) synthesis of brush copolymers by ATRP.

\section{Experimental}

\subsection{General information}

Chemicals were purchased from Sigma-Aldrich, VWR or Fisher Scientific and used without further purification. Solvents used in reactions were from alumina filtration system. 2-(2bromoisobutyryloxy)ethyl methacrylate (BIEM) was synthesized according to literature [28]. Acrylate amide monomer 5-acetylaminopentyl acrylate (AAPA) was synthesized as reported [22]. All glassware was flame dried before use, and reactions were carried out under nitrogen atmosphere. Extraction solvents were commercial grade. Flash Chromatography was performed using forced flow of the indicated solvent systems over Fisher silica gel (230 - 400 mesh). ${ }^{1} \mathrm{H}$ NMR spectra were taken on a $500 \mathrm{MHz}$ Bruker instrument. Gel permeation chromatography (GPC) was performed in THF (1 mL/min) using an Agilent LC 1100 Series equipped with a 
Polymer Laboratory's PLgel $5 \mu \mathrm{m}$ mixed-C column to determine molecular weights and molecular weight distributions (PDI), $M_{\mathrm{w}} / M_{\mathrm{n}}$, of the macroinitiators with respect to polymethylmethacrylate (PMMA) standards (Agilent Technologies, Inc.). Molecular weights of brushed polymers were determined by size-exclusion chromatography (SEC) in DMF (1.0 $\mathrm{mL} / \mathrm{min}$ ) coupled with a Dawn DSP18 multi-angle light scattering detector (MALS, laser wavelength $\lambda=632 \mathrm{~nm}$, Wyatt Technology, Santa Babara, CA) and a Refractometric Index (RI) detector (an Agilent 1100 SEC system with a ShodexOHpak SB 803-HQ column). The hydrodynamic radius $\left(R_{\mathrm{h}}\right)$ of the polymers in a DMF or methanol solution were measured by Dynamic Light Scattering (DLS) using a Zetasizer Nano Series (Malvern Instrument, Model: ZEN3600) at $25^{\circ} \mathrm{C}$.

\subsection{Synthesis of macroinitiators}

The macroinitiators were synthesized by free-radical polymerization (Scheme 1a). As an example, the synthesis for Macroinitiator 1 is described here. Methylmethacrylate (MMA) (3.6043 g, $36 \mathrm{mmol}), 2$-(2-bromoisobutyryloxy)ethyl methacrylate (BIEM) (1.1165 g, $4 \mathrm{mmol})$ and AIBN (16.4 mg, $0.1 \mathrm{mmol}$ ) were dissolved in $20 \mathrm{~mL}$ toluene. The mixture was stirred at room temperature for $30 \mathrm{~min}$, and then was heated at $70{ }^{\circ} \mathrm{C}$ for $24 \mathrm{~h}$ under nitrogen. Upon cooling to room temperature, the polymer was precipitated in methanol, filtered and washed thoroughly with methanol, and then dried at $60{ }^{\circ} \mathrm{C}$ under vacuum for $24 \mathrm{~h}$ to give $3.7031 \mathrm{~g}(78 \%$ yield) Macroinitiator 1 as a white powder. GPC: $M_{\mathrm{n}}, 34.2 \mathrm{~kg} / \mathrm{mol}$; PDI, 1.90 .

Macroinitiator 2 was prepared in the same manner with $3.8046 \mathrm{~g}(38 \mathrm{mmol})$ of MMA, $0.5583 \mathrm{~g}$ ( $2 \mathrm{mmol})$ of BIEM and $16.4 \mathrm{mg}(0.1 \mathrm{mmol})$ of AIBN. GPC: $M_{\mathrm{n}}, 35.9 \mathrm{~kg} / \mathrm{mol} ;$ PDI, 1.79 .

\subsection{Synthesis of brush copolymers}


The brush copolymers were synthesized by ATRP polymerization. As an example, the synthesis of Brush 1 is described here. Macroinitiator 1 (0.236 g, $0.2 \mathrm{mmol} \mathrm{Br})$, 5-acetylaminopentyl acrylate (AAPA) (9.963 g, $50 \mathrm{mmol})$, and PMDETA $(69.3 \mathrm{mg}, 0.4 \mathrm{mmol})$ were dissolved in DMF $(25 \mathrm{~mL})$. The mixture was degassed by three freeze-pump-thaw cycles. After addition of $\mathrm{CuBr}(28.7 \mathrm{mg}, 0.2 \mathrm{mmol})$, the mixture was heated at $70{ }^{\circ} \mathrm{C}$ for $24 \mathrm{~h}$ under stirring. The monomer conversion was determined by ${ }^{1} \mathrm{H}$ NMR spectroscopy of the polymerization mixture. Upon cooling, the polymer was precipitated and re-precipitated three times in ether to thoroughly remove any residual monomer. The copper catalyst was removed by passing a solution of the polymer in methanol over a basic alumina plug. The dried polymer $(7.48 \mathrm{~g})$ was obtained by evaporating the methanol solution under vacuum at $110{ }^{\circ} \mathrm{C}$ for $48 \mathrm{~h}$ (SEC-MALS: $M_{\mathrm{n}}, 1460$ kg/mol; PDI, 1.85).

Brush 2, 3 and 4 were prepared by ATRP polymerization using Macroinitiator 2 as the initiator. For Brush 2, $1.0907 \mathrm{~g}$ (0.5 mmol Br) of Macroinitiator 2, $9.9625 \mathrm{~g}$ (50 mmol) of AAPA, 173.3 $\mathrm{mg}(1 \mathrm{mmol})$ of PMDETA and $71.7 \mathrm{mg}(0.5 \mathrm{mmol})$ of $\mathrm{CuBr}$ were used. After purification, $7.80 \mathrm{~g}$ of Brush 2 were obtained (SEC-MALS: $M_{\mathrm{n}}, 349 \mathrm{~kg} / \mathrm{mol}$; PDI, 1.71).

For Brush 3, $0.4363 \mathrm{~g}(0.2 \mathrm{mmol} \mathrm{Br})$ of Macroinitiator 2, $9.9625 \mathrm{~g}$ (50 mmol) of AAPA, 69.3 $\mathrm{mg}(0.4 \mathrm{mmol})$ of PMDETA and $28.7 \mathrm{mg}(0.2 \mathrm{mmol})$ of $\mathrm{CuBr}$ were used. After purification, $6.81 \mathrm{~g}$ of Brush 3 were obtained (SEC-MALS: $M_{\mathrm{n}}, 715 \mathrm{~kg} / \mathrm{mol}$; PDI, 1.60).

For Brush 4, $0.238 \mathrm{~g}(0.11 \mathrm{mmol} \mathrm{Br})$ of Macroinitiator 2, $9.9625 \mathrm{~g}$ (50 mmol) of AAPA, 69.3 $\mathrm{mg}(0.4 \mathrm{mmol})$ of PMDETA and $28.7 \mathrm{mg}(0.2 \mathrm{mmol})$ of $\mathrm{CuBr}$ were used. After purification, $6.64 \mathrm{~g}$ of Brush 4 were obtained (SEC-MALS: $M_{\mathrm{n}}, 1140 \mathrm{~kg} / \mathrm{mol}$; PDI, 1.56). 


\subsection{AFM studies}

Atomic force microscopy (AFM) was performed on a MFP-3D-SA AFM instrument (Asylum Research, Santa Barbara, CA) using silicon tips (type AC240TS) with a spring constant of 2 N/m at a resonance frequency of $70 \mathrm{kHz}$. All measurements were carried out in tapping (AC) mode. The samples were spin-coated from solution $(1 \mathrm{mg} / \mathrm{mL})$ on mica substrates.

\subsection{Transmission electron microscopy (TEM) studies}

TEM was performed on a FEI/Philips CM-20 conventional TEM operated at an accelerating voltage of $200 \mathrm{kV}$. The sample was stained by floating the TEM grid on a $0.5 \mathrm{wt} \%$ aqueous solution of uranyl acetate for 1 minute, followed by removing excess solvent by placing the sample on filter paper.

\subsection{Differential scanning calorimetry (DSC) and thermogravimetric analysis (TGA)}

DSC was performed on a TA Instruments Q2000 using standard heat-cool-heat experiments on $\sim 5 \mathrm{mg}$ of sample between -80 and $200{ }^{\circ} \mathrm{C}$. The heating rate was $10{ }^{\circ} \mathrm{C} / \mathrm{min}$. The glass transition temperatures $\left(T_{g}\right)$ were reported at the inflection point of the heat capacity jump of the second heating run. TGA was performed on a TA Instruments Q500. Samples of $\sim 5 \mathrm{mg}$ were heated from 30 to $850{ }^{\circ} \mathrm{C}$ under nitrogen at a rate of $20^{\circ} \mathrm{C} / \mathrm{min}$.

\subsection{Mechanical studies}

Storage moduli of the brush copolymers were obtained by the time sweep experiment on a rheometer (AR-G2 from TA Instruments) equipped with $20 \mathrm{~mm}$ parallel plates at the conditions of $1 \%$ strain, $1 \mathrm{~Hz}$ and $20{ }^{\circ} \mathrm{C}$. Creep experiments were performed on a dynamic mechanical 
analyzer (DMA Q800 from TA Instruments). Mechanical tensile testing and stress-relaxation experiments were performed on an Instron 3365 machine. Samples were prepared by hotpressing the resin into Teflon molds (30 mm x $7 \mathrm{~mm} \times 2 \mathrm{~mm}$ ). The specimens were extended at $100 \mathrm{~mm} / \mathrm{min}$ at room temperature. Each measurement was repeated at least three times. Young's modulus $(E)$ was determined from the initial slope of the stress-strain curves. Self-healing specimens were prepared by cutting the film in half, gently pushing two interfaces together for a minute and letting the film heal at room temperature for a certain time.

\section{Results and discussion}

As shown in Scheme 1a, the macroinitiators for ATRP were prepared by free radical copolymerization of methyl methacrylate (MMA) and 2-(2-bromoisobutyryloxy)ethyl methacrylate (BIEM). We controlled the densities of the grafting side chains by adjusting the molar ratios of BIEM to MMA (1 : 9 and $1: 19$ for Macroinitiator 1 and Macroinitiator 2, respectively). The free radical polymerization was conducted at $70{ }^{\circ} \mathrm{C}$ for 24 hours. We used relatively low concentration of AIBN initiator for obtaining relatively high molecular weight of the macroinitiators. Macroinitiator 1 and 2 contained $10 \mathrm{~mol} \%$ and $5 \mathrm{~mol} \%$ of BIEM moieties, respectively, which was confirmed by ${ }^{1} \mathrm{H}-\mathrm{NMR}$ spectroscopy (see Supplemental Data, Fig. S1 and Fig S2). Molecular weights were determined by GPC using PMMA standards. Macroinitiator1 had a molecular weight $\left(M_{\mathrm{n}}\right)$ of $34.2 \mathrm{~kg} / \mathrm{mol}$ with a polydispersity (PDI) of 1.90, which was estimated to have $29 \mathrm{Br} / \mathrm{chain}$. The $M_{\mathrm{n}}$ and PDI for Macroinitiator 2 were 35.9 $\mathrm{kg} / \mathrm{mol}$ and 1.79 , respectively, with $16 \mathrm{Br} /$ chain. 
ATRP polymerization was performed to attach PA-amide to the PMMA backbone (Scheme 1b). The polymerization results were summarized in Table 1. The brush lengths of the brush copolymers were tailored by adjusting the molar ratios of AAPA to $\mathrm{Br}$ in the macroinitiator during graft copolymerization. The brush length was estimated by the conversion of the monomers using ${ }^{1} \mathrm{H}-\mathrm{NMR}$ technique. The overall molecular weights of the brush copolymers were determined by SEC with multi-angle light scattering (MALS) measurement. As showed in Table 1, the molecular weights determined by MALS were in good agreement with those estimated by conversion based on ${ }^{1} \mathrm{H}-\mathrm{NMR}$ spectra. The brush lengths of Brush $\mathbf{1}$ was 227 , using Macrointitiator 1 as the initiator. The brush lengths of Brush 2, 3, and 4 were 88, 222 and 324, respectively, synthesized from Macroinitiator 2. All of the brush copolymers had high molecular weights $\left(M_{\mathrm{n}}\right)$ ranging from $0.3-1.3 \times 10^{3} \mathrm{~kg} / \mathrm{mol}$ (Table 1$)$.

Table 1 The polymerization results of ATRP for brush copolymers.

\begin{tabular}{ccccccc}
\hline & $\begin{array}{c}\text { AAPA: } \\
\mathrm{Br}^{\mathrm{a}}\end{array}$ & $\begin{array}{c}\text { Conversion, } \\
\%\end{array}$ & $\begin{array}{c}\text { Brush } \\
\text { length }\end{array}$ & $\begin{array}{c}M_{\mathrm{n}}{ }^{\mathrm{b}}, \\
\mathrm{kg} / \mathrm{mol}\end{array}$ & $\begin{array}{c}M_{\mathrm{n}}{ }^{\mathrm{c}}, \\
\mathrm{kg} / \mathrm{mol}\end{array}$ & $\mathrm{PDI}^{\mathrm{d}}$ \\
\hline Brush 1 & $250^{\mathrm{e}}$ & 91 & 227 & 1,346 & 1,460 & 1.85 \\
Brush 2 & $100^{f}$ & 88 & 88 & 325 & 349 & 1.71 \\
Brush 3 & $250^{f}$ & 89 & 222 & 767 & 715 & 1.60 \\
Brush 4 & $463^{\mathrm{f}}$ & 70 & 324 & 1,101 & 1,140 & 1.56 \\
\hline
\end{tabular}

a) Molar ratio of monomer AAPA to $\mathrm{Br}$ for ATRP polymerization. b) Molecular weight estimated by conversion determined by ${ }^{1} \mathrm{H}$ NMR. c) Molecular weight determined by SECMALS. d) Polydispersity determined by SEC-MALS. e) Macroinitiator 1 was used as the initiator. f) Macroinitiator 2 was used as the initiator. 
Based on our previous work [22], we envisioned that the brush copolymers should adopt spherical topology in solution that would further self-assemble to form spherical particles in solid state. The solution morphology was confirmed by both static and dynamic light scattering studies. The radius of gyration $\left(R_{\mathrm{g}}\right)$ was determined by MALS with a polymer solution of 1 $\mathrm{mg} / \mathrm{mL}$ in dimethylformamide (DMF). The hydrodynamic radius $\left(R_{\mathrm{h}}\right)$ of the brush copolymers was measured by dynamic light scattering (DLS) of in the same solution. The ratios of $R_{\mathrm{g}} / R_{\mathrm{h}}$ for the brush copolymers Brush 1 is 1.1, implying an extended coiled structure formed in DMF solution, which was expectable, since DMF was good solvent for both PMMA and PA-amide. Surprisingly, reducing the density and brush length, Brush 2 and $\mathbf{3}$ adopted spherical morphology in DMF solution with $R_{\mathrm{g}} / R_{\mathrm{h}}$ ratio of 0.7 and 0.5 , respectively [29]. Brush 4 could not provide reliable DLS measurement in DMF, but in $1 \mathrm{mg} / \mathrm{mL}$ methanol solution, the $R_{\mathrm{h}}$ was $40 \mathrm{~nm}$. The spherical morphology was further verified by TEM and AFM imaging in solid state of the individual nanoparticles. Brush 2 was deposited on the TEM grid by dip coating and stained by uranyl acetate ( $0.5 \mathrm{wt} \%$ solution in water). The diameter of Brush 2 in dry state was about $30 \mathrm{~nm}$ by TEM measurement (Fig. 1a and Fig. S7 in Supplemental Data). AFM measurement gave a larger particle diameter (about $40 \mathrm{~nm}$ ) than that observed by TEM (Fig. 1b). The discrepancy was attributed to the tip convolution effect associated with AFM scanning. 
a

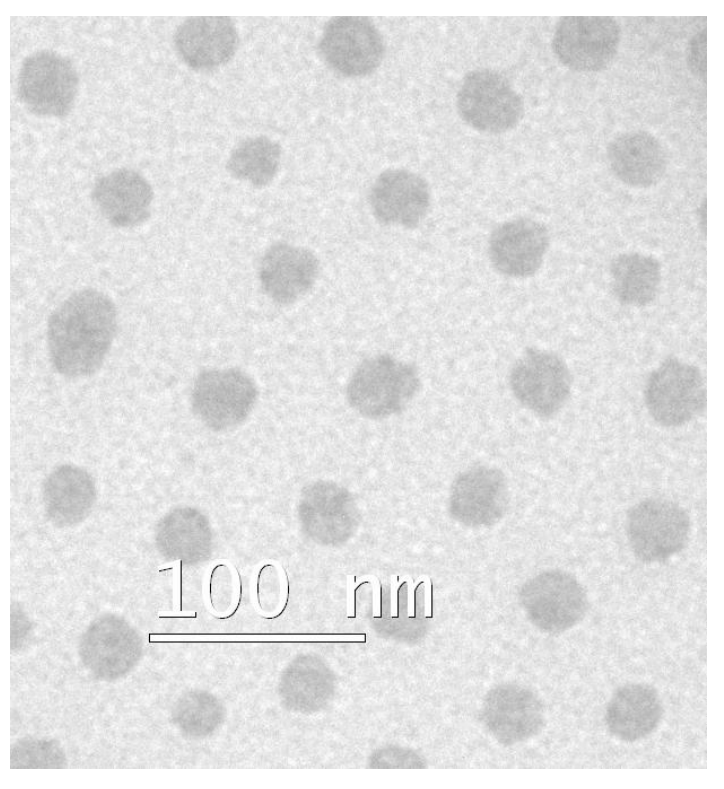

b
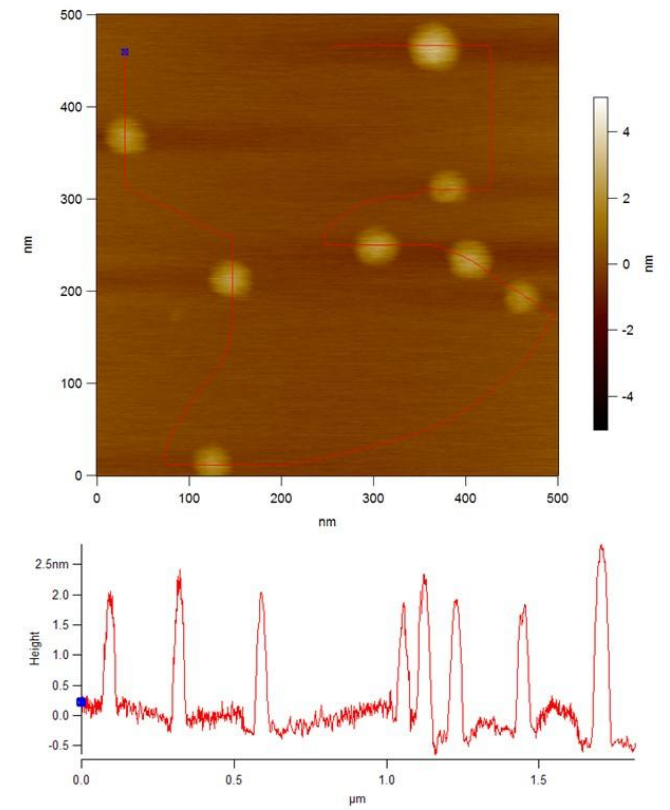

Figure 1. Morphology study of Brush 2. a) TEM image of Brush 2. b) AFM image of Brush 2.

The thermal properties of the brush copolymers were investigated by DSC and TGA. The detectable glass transition temperature $\left(T_{\mathrm{g}}\right)$ of brush copolymers was below room temperature $\left(1 \sim 5{ }^{\circ} \mathrm{C}\right)$, which was attributed to the soft PA-amide brushes (Table 2) [22]. The brush copolymers have reasonably good thermal stability. A determined by the thermal gravimetric analysis (TGA) under nitrogen atmosphere, the sample lost $5 \%$ weight $~ 320^{\circ} \mathrm{C}$ (Table 2 , and Fig. S8). 
Table 2 Characterization of brush copolymers.

\begin{tabular}{|c|c|c|c|c|c|c|c|c|c|}
\hline & $\begin{array}{l}\mathrm{R}_{\mathrm{g}}{ }^{2} \\
\mathrm{~nm}\end{array}$ & $\begin{array}{l}\mathrm{R}_{\mathrm{h}}{ }^{\prime} \\
\mathrm{nm}\end{array}$ & $\mathrm{R}_{\mathrm{g}} / \mathrm{R}_{\mathrm{h}}$ & $\begin{array}{l}\mathrm{Tg}^{\mathrm{c}} \\
{ }^{\circ} \mathrm{C}\end{array}$ & $\begin{array}{c}5 \% \\
\text { weight } \\
\text { lostd },{ }^{\circ} \mathrm{C}\end{array}$ & $\begin{array}{c}\text { Young's } \\
\text { modulus, } \\
\mathrm{MPa}\end{array}$ & $\begin{array}{c}\text { Yield } \\
\text { strengthe } \\
\mathrm{MPa}\end{array}$ & $\begin{array}{c}\text { Breaking } \\
\text { strength, } \\
\mathrm{MPa}\end{array}$ & $\begin{array}{c}\text { Breaking } \\
\text { strain, } \\
\mathrm{mm} / \mathrm{mm}\end{array}$ \\
\hline Brush 1 & 27 & 24 & 1.1 & 2 & 329 & $11.7 \pm 1.0$ & $0.30 \pm 0.01$ & $0.64 \pm 0.02$ & $-\mathrm{g}$ \\
\hline Brush 2 & 22 & 32 & 0.7 & 5 & 316 & $32.2 \pm 1.4$ & $0.80 \pm 0.03$ & $3.08 \pm 0.07$ & $1.9 \pm 0.1$ \\
\hline Brush 3 & 18 & 40 & 0.5 & 5 & 326 & $17.1 \pm 1.3$ & $0.46 \pm 0.04$ & $2.43 \pm 0.09$ & $5.1 \pm 0.5$ \\
\hline Brush 4 & 26 & $-f$ & - & 1 & 328 & $15.9 \pm 0.9$ & $0.39 \pm 0.03$ & $2.00 \pm 0.04$ & $10.9 \pm 0.8$ \\
\hline
\end{tabular}

a) Determined by MALS. b) Determined by DLS. c) Determined by the second scan of DSC measurement. d) The temperature at which the sample lost 5\% weight, which was determined by TGA. e) Taken as strength at $10 \%$ strain. f) Not available due to poor data quality. g) Not broken at $2000 \%$ strain.

The mechanical properties of the brush copolymers were evaluated by the tensile test. Specimens were prepared by hot-pressing the synthesized brush copolymer resins into an array of Teflon molds (30 mm x $7 \mathrm{~mm} \times 2 \mathrm{~mm}$ ). The stress-strain curves for the four brush copolymer samples were compared in Figure 2. Both the density and the length of the PA-amide brushes were varied to tune mechanical properties of the brush copolymers. First, at constant PMMA backbone and PA-amide brush length, decreasing the brush density significantly impact the mechanical properties of the materials. With similar brush length ( 200 repeating units per brush), Brush 1 did not break at a strain of $2000 \%$, while Brush 3 broke at a strain of $500 \%$. However, Brush 3 has higher Young's modulus and tensile strength. The ultimate tensile strength was $\sim 2.5 \mathrm{MPa}$ for Brush 3, while the value was $0.6 \mathrm{MPa}$ for Brush 1 (Table 2 and Fig. 2). Next, the brush length was altered to tune the mechanical properties of the materials. Brush $\mathbf{2}, \mathbf{3}$, and $\mathbf{4}$ were all generated from Macroinitiator 2, but had different brush length with repeating units of 88, 222 and 324, respectively. The materials became stiffer with decreasing brush length, as shown by the increasing values of the Young's modulus (32.2, 17.1 and 15.9 MPa for Brush 2, 3, and 4, 
respectively) and the tensile strength (3.08, 2.43 and 2.00 MPa for Brush 2, 3, and 4, respectively) (Table 2 and Fig. 2). Overall, an increase of the volume fraction of the hard PMMA phase is correlated with stronger materials with higher Young's modulus and tensile strength. On the contrary, an increase of the volume fraction of the PA-amide soft phase is correlated with less stiff but more elastomeric materials with larger strain-at-break. This trend was also observed in the rheological testing. In the time sweep experiment, the storage modulus slight increased from 2.0 to 2.3 MPa for Brush 4 and 3, but jumped to 4.6 MPa for Brush 2 (Fig. S9 in Supplemental Data).

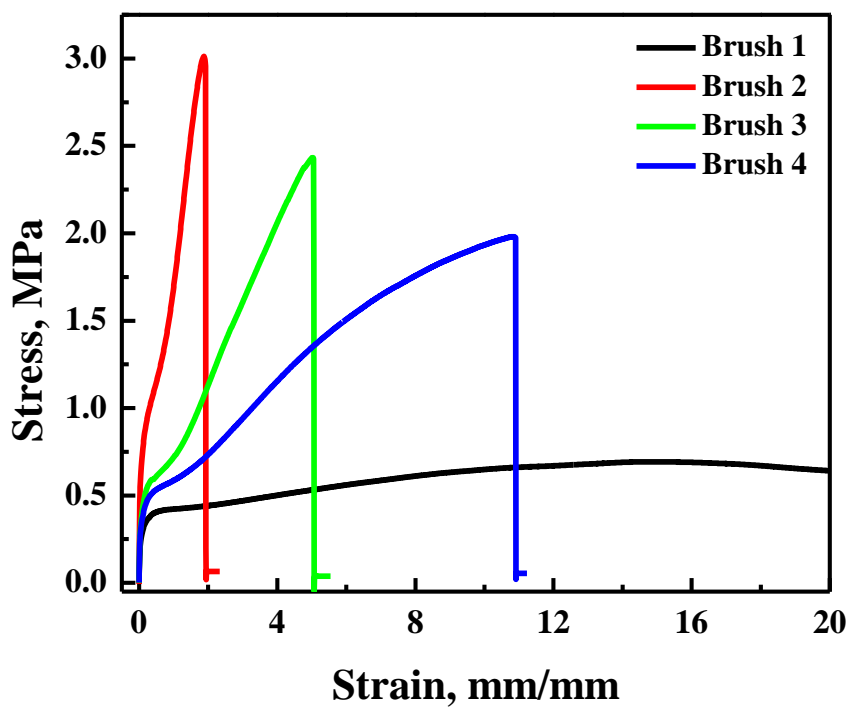

Figure 2. The stress-strain curves of brush copolymers. The strain rate was $100 \mathrm{~mm} / \mathrm{min}$.

The thermoplastic elastomer properties were further confirmed by creep-recovery and stressrelaxation experiments of Brush 2 (Fig. 3). At $23{ }^{\circ} \mathrm{C}$, a stress of 4 x $10^{4} \mathrm{~Pa}$ applied to Brush 2 for 600 minutes results in a strain of about $31 \%$ that increases at a rate of $0.19 \%$ per hour. After releasing the applied stress, the sample completely recovers its dimensions with negligible 
residual strain $(<1 \%)$. Applying a higher stress $\left(8 \times 10^{4} \mathrm{~Pa}\right)$ for the same amount of time leads to a strain of about $63 \%$ that increases by $0.98 \%$ per hour. Release of the stress leaves a residual strain of less than $1 \%$ (Fig. 3a). The tensile stress relaxation experiments further confirm the elastomeric properties of the brush copolymer (Fig. 3b). Different from conventional TPEs that normally have rubbery chains covalently linked between hard domains, in our system the PMMA hard domains are connected by multivalent hydrogen bonds between the soft PA-amide brushes. Despite the supramolecular nature of such dynamic interactions, the multivalent hydrogen bonding networks provide sufficient cohesive energy to form robust TPE materials.

a

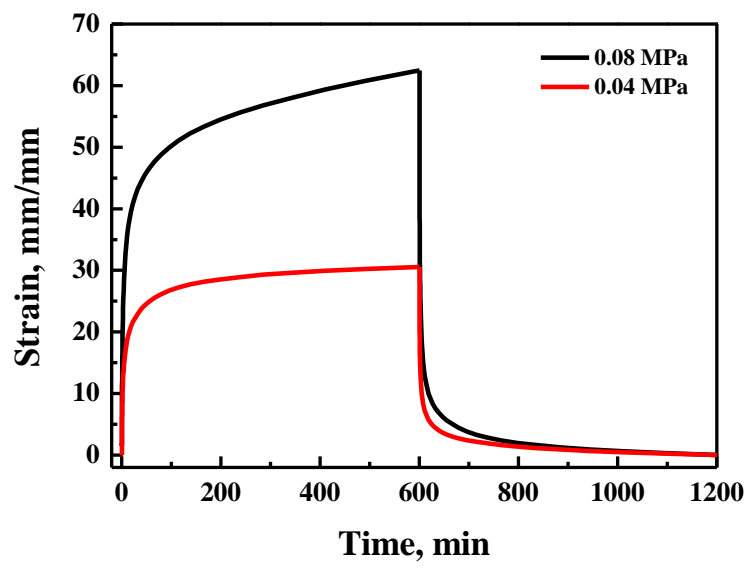

b

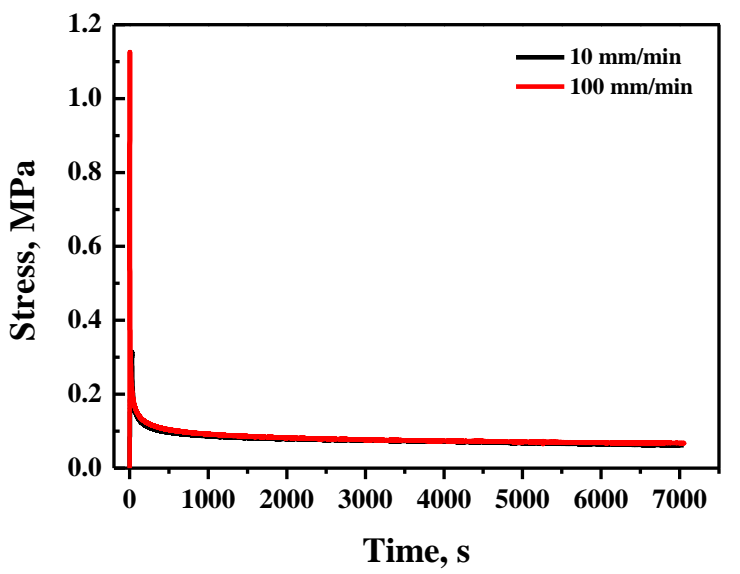

Figure 3. The creep-recovery and stress-relaxation behavior of Brush 2. a) The creep-recovery result. The film was pulled at a certain stress for $600 \mathrm{~min}$, and then the stress was released and the films were recovered for another $600 \mathrm{~min}$ at $23{ }^{\circ} \mathrm{C}$. b) The stress-relaxation of Brush 2 . The sample was pulled at a rate of 10 or $100 \mathrm{~mm} / \mathrm{min}$ to reach a $100 \%$ strain, which was set at this strain for relaxation for 120 minutes. 
a.

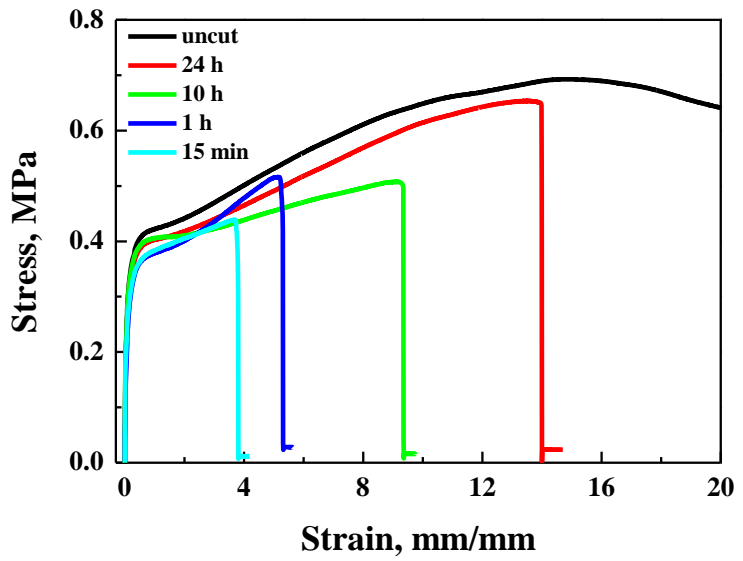

C.

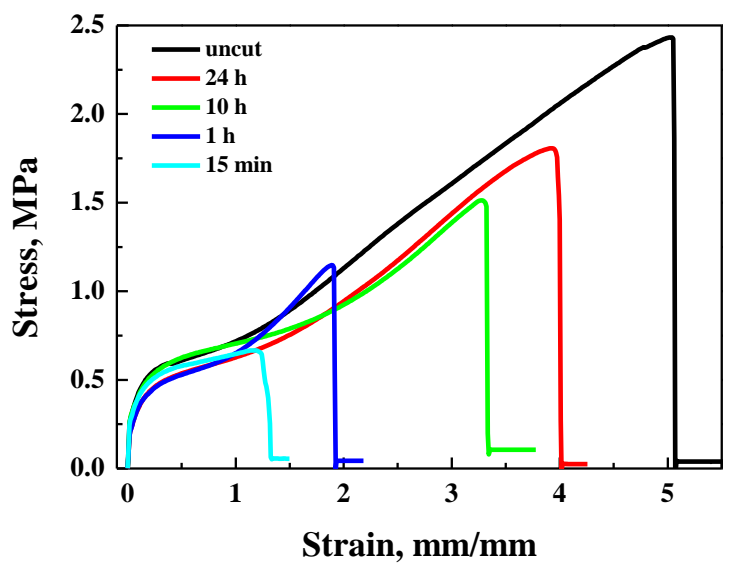

b.

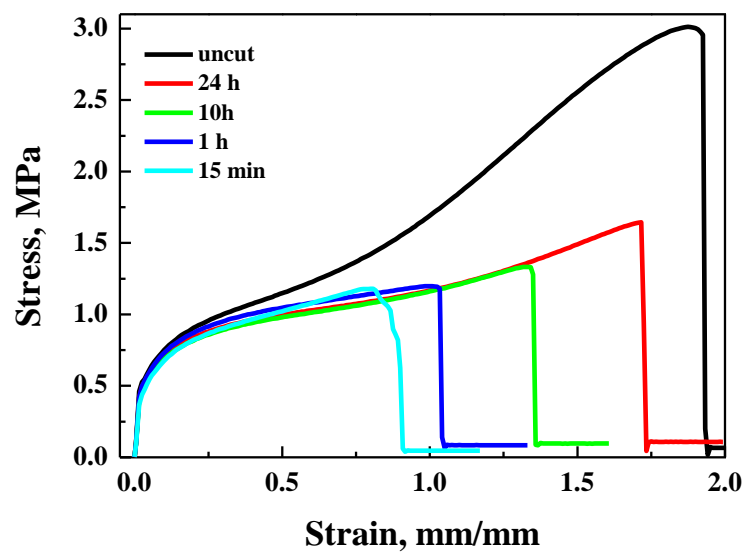

d.

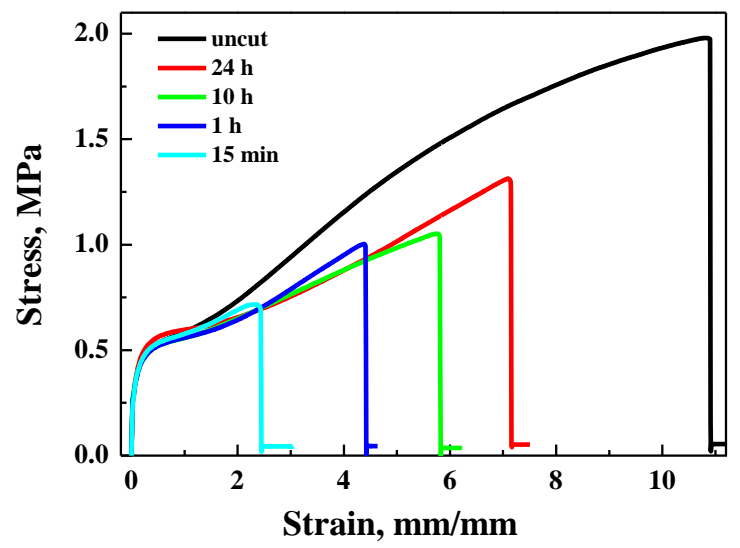

Figure 4. The self-healing properties of the brush copolymers. The strain rate was $100 \mathrm{~mm} / \mathrm{min}$. a) Brush 1. b) Brush 2. c) Brush 3. d) Brush 4.

Inherent to the molecular design, the $T_{g}$ of the PA-amide brushes is relatively low $\left(1-5{ }^{\circ} \mathrm{C}\right.$, Table 2), which would provide high mobility for the brushes at ambient temperature. As demonstrated in several previous studies [22, 25, 27], the dynamic hydrogen bonding interactions between secondary amide groups in the soft PA-amide phase can effectively heal samples after mechanical damage. We investigated the self-healing properties of the current brush copolymer samples by following the same protocols used previously. After cutting the sample specimens into half, the two cut surfaces were gently pushed together for a minute and 
then left at ambient temperature to heal for a certain time. As shown in Figure 4, the materials could recover the yield strength within 15 minutes, and the healing improved with increasing healing time. The stress-strain curves for the healed specimens followed closely the curves of their pristine specimens. After 24-hour healing, the strain recovery for all samples passed $60 \%$ (Fig. 4). The total recovered strains for Brush 2 and $\mathbf{3}$ were $\sim 80 \%$ after 24-hour healing (Fig. 4b and 4c), with Brush 1 healed specimen ruptured at an impressive strain of $~ 1400 \%$ (Fig. 4a). Overall, the materials showed relatively good self-healing capability in term of both strength and extensibility.

\section{Conclusion}

In summary, self-healing brush copolymers with a glassy PMMA backbone and rubbery PAamide brushes have been prepared in two-step polymerization. The PMMA macroinitiators containing multiple ATRP initiators (BIEM) were prepared by free radical polymerization, followed by subsequent ATRP to generate the brush copolymers. The brush copolymers adopted spherical morphology both in solution and in dried state. In bulk materials, the materials showed

thermoplastic elastomer type mechanical properties. Importantly, the PA-amide brushes of the brush copolymers were highly mobile and rich in dynamic hydrogen bonding amide functional groups, which bestowed the self-healing properties to the materials at room temperature without the need of any external stimulus. 


\section{Acknowledgements}

We acknowledge the financial support from the US Department of Energy, Division of Materials

Science (award no. DE-FG02-04ER46162). Y. C. acknowledges the UC Regent's Dissertation Fellowship.

\section{Appendix A. Supplementary data}

${ }^{1} \mathrm{H}$ NMR spectra of macroinitiators and brush copolymers, additional TEM images of Brush $\mathbf{2}$, TGA trace of Brush 2 and Storage moduli of brush copolymers of this article can be found at:

\section{References}

[1] Wu D, Meuer S, Solomon D. Prog Polym Sci 2008; 33: 479-522.

[2] Murphy EB, Wudl F. Prog Polym Sci 2010; 35: 223-251.

[3] Blaiszik BJ, Kramer SLB, Olugebefola SC, Moore JS, Sottos NR, White SR. Annu Rev Mater Res 2010; 40: 179-211.

[4] Burattini S, Greenland BW, ChappellD, Colquhoun HM, Hayes W. Chem Soc Rev 2010; 39: 1973-1985.

[5] Syrett JA, Becer CR, Haddleton DM. Polym Chem 2010; 1: 978-987.

[6] White SR, Sottos NR, Geubelle PH, Moore JS, Kessler MR, Sriram SR, Brown EN, Viswanathan S. Nature 2001; 409: 794-797.

[7] White SR, Caruso MM, Moore JS. MRS Bulletin 2008; 33: 766-769.

[8] Chen X, Dam MA, Ono K, Mal A, Shen H, Nutt SR, Sheran K, Wudl F. Science 
2002; 295: 1698-1702.

[9] Chung C, Roh Y, Cho S, Kim J. Chem Mater 2004; 16: 3982-3984.

[10] Amamoto Y, Kamada J, Otsuka H, Takahara A, Matyjaszewski K. Angew Chem Int Ed 2011; 50: 1660-1663.

[11] Imato K, Nishihara M, Kanehara T, Amamoto Y, Takahara A, Otsuka H. Angew Chem Int Ed 2012; 51: 1138-1142.

[12] Lu Y, Guan Z. J Am Chem Soc 2012; 134: 14226-14231.

[13] Ghosh B, Urban MW. Science 2009; 323: 1458-1460.

[14] Ghosh B, Chellappan KV, Urban MW. J Mater Chem 2011; 21: 14473-14486.

[15] Burnworth M, Tang L, Kumpfer JR, Duncan AJ, Beyer FL, Fiore GL, Rowan SJ, Weder C. Nature 2011; 472: 334-338.

[16] Mozhdehi D, Ayala S, Cromwell OR, Guan Z. J Am Chem Soc 2014; 136: 16128-16131.

[17] Burattini S, Colquhoun HM, Fox JD, Friedmann D, Greenland BW, Harris PJF, Hayes W, Mackay ME, Rowan SJ. Chem Commun 2009; 6717-6719.

[18] Burattini S, Greenland BW, Merino DH, Weng W, Seppala J, Colquhoun HM, Hayes W, Mackay ME, Hamley IW, Rowan SJ. J Am Chem Soc 2010; 132: 12051-12058.

[19] Cordier P, Tournilhac F, Soulie-Ziakovic C, Leibler L. Nature 2008; 451: 977-980.

[20] Folmer BJB, Sijbesma RP, Versteegen RM, van der Rijt JAJ, Meijer EW Adv Mater 2000; 12: $874-878$.

[21] Wang C, Liu N, Allen R, Tok JBH, Wu Y, Zhang F, Chen Y, Bao Z. Adv Mater 2013; 25: $5785-5790$.

[22] Chen Y, Kushner AM, Williams GA, Guan Z. Nat Chem 2012; 4: 467-472.

[23] Wojtecki RJ, Meador MA, Rowan SJ. Nat Mater 2010; 10: 14-27.

[24] Hoogenboom R. Angew Chem Int Ed 2012; 51: 11942-11944. 
[25] Chen Y, Guan Z. Polym Chem 2013; 4: 4885-4889.

[26] Hentschel J, Kushner AM, Ziller J, Guan Z. Angew Chem Int Ed 2012; 51: 10561-10565.

[27] Chen Y, Guan Z. Chem Commun 2014; 50: 10868-10870.

[28] Matyjaszewski K, Pyun J, Gaynor SG. Macromol Rapid Commun 1998; 19: 665-670.

[29] Burchard W. Static and dynamic light scattering from branched polymers and biopolymers, in light Scattering from Polymers Vol. 48, 1-24 (Springer 1983). 
- Brush copolymers with PMMA backbone and multiple flexible PA-amide brushes carrying multivalent hydrogen bonds were prepared.

- The brush copolymers were able to self-assemble into spherical nanoparticles.

- The brush copolymers behaved like thermoplastic elastomers with tunable mechanical properties due to microphase separation.

- The brush copolymers show a combination of strong mechanical properties and selfhealing properties due to our multiphase design. 


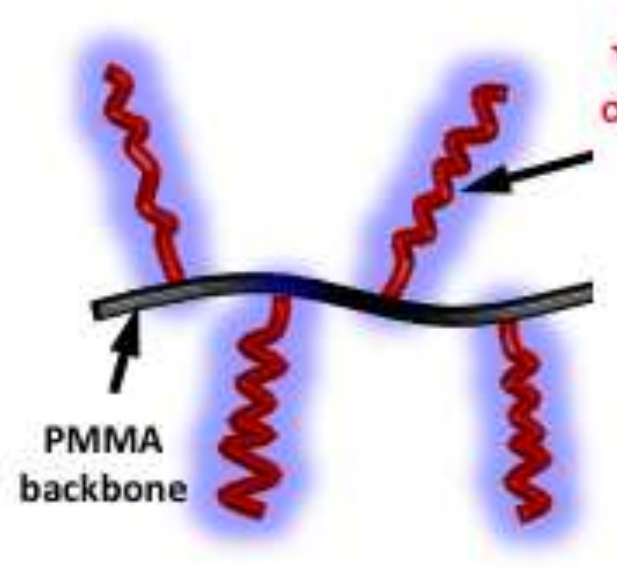

Brush copolymers

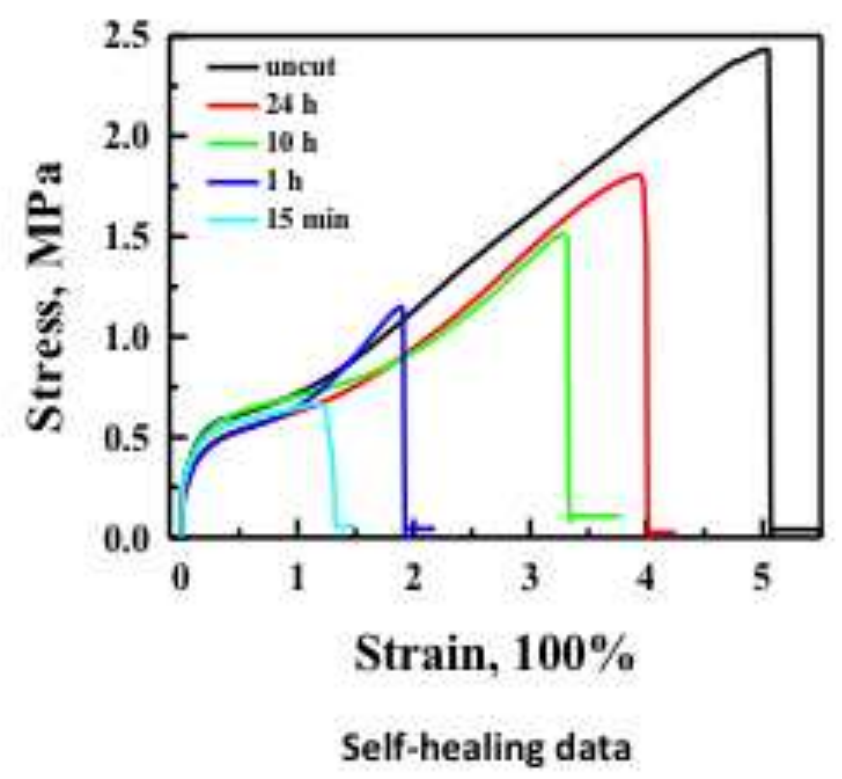

AFM image
Self-healing data 